\title{
CORRELATION OF ANXIETY DISORDER AND QUALITY OF LIFE IN SYSTEMIC
}

\section{LUPUS ERYTHEMATOSUS PATIENTS}

\section{Kemala Emrizal ${ }^{1}$, Lynna Lidyana ${ }^{2}$, Laniyati Hamijoyo ${ }^{3,4}$}

${ }^{1}$ Faculty of Medicine Padjadjaran University, Bandung, Indonesia

2Department of Psychiatry, Faculty of Medicine Padjadjaran University/Dr. Hasan Sadikin Hospital, Bandung, Indonesia

${ }^{3}$ Department of Internal Medicine, Rheumatology Division, Faculty of Medicine, Padjadjaran University/Dr. Hasan Sadikin

Hospital, Bandung, Indonesia

${ }^{4}$ Lupus Study Group, Immunology Research Center, Faculty of Medicine, Padjadjaran University, Bandung, Indonesia

\section{A R T I C L E I N F O \\ Keywords: \\ Anxiety Disorder \\ Quality of Life \\ Systemic Lupus Erythematosus \\ Zung Self Rating Anxiety Scale.}

Corresponding author:

Laniyati Hamijoyo

E-mail address:

hamijoyo@yahoo.com

All authors have reviewed and approved the final version of the manuscript.

https://doi.org/10.37275/IJR.v10i1.1

\begin{abstract}
A B S T R A C T
Background: Systemic Lupus Erythematosus (SLE), an autoimmune disease, can cause damage and impairment in the nervous system. Patients who had any manifestation of neurology can be classified as patients with Neuropsychiatric Systemic Lupus Erythematosus (NPSLE). One of the most frequent NPSLE manifestation is anxiety disorder. The presence of anxiety disorder is believed to be correlated with their ability to carry out daily activities. This study aims to see the correlation between anxiety disorder and quality of life (QOL) in patients with SLE. Method: an analitic cross-sectional study was done. The data were collected by distributing validated questionnaires to patients diagnosed with SLE in the outpatient clinic of dr. Hasan Sadikin General Hospital. Quality of life and anxiety disorder was measured using Short From-36 (SF-36) and Zung Self-Rating Anxiety Scale (ZungSAS), respectively. Normality test was done before correlating the variables using Pearson method. Result: Forty-six SLE patients fitted with the inclusion criteria were participated in the study. The assessment using Zung-SAS showed that $9(19.56 \%)$ correspondents had mild-moderate anxiety, and $1(2.17 \%)$ had severe anxiety. The analysis of SF-36 showed the means of Physical Component Summary (PCS) and Mental Component Summary (MCS) which were 45.18 \pm 8.23 and 47.11 \pm 9.78 , in order. The correlation test of Zung-SAS with PCS and MCS showed the result of $r=-$ $0.651(p<0,01)$ and $-0.654(p<0,01)$, respectively. Conclusion: There is a significant negative correlation between anxiety disorder and QOL in patients with SLE. The result of this study showed that the high degree of ones anxiety was in a parallel line with their low level of QOL, so it is important to do an early detection and prevention of anxiety disorder in SLE patients.
\end{abstract}

\section{Introduction}

Systemic Lupus Erythematosus (SLE) is an autoimmune disease causing damages in various body organs. Lupus is categorized as a systemic and chronic disease. Skin and mucous, joints, blood, heart, lungs, nervous system, and immune system could all be involved in the course of the disease. The chronic symptoms of SLE, such as joint pain and fatigue may disturb the daily activity of patients, therefore influencing their quality of life (QOL). The long nature of lupus disease course can lead into the increase of patients' mental problem, such as emotional disturbance, anxiety disorder, even depression.1,2 Systemic Lupus Erythematosus in the countries of Asia-Pacific had a crude incidence rate (per $100.000 /$ year) of $0,9-3,1$ and crude prevalence rates of 4,3-45,3 (per 100.000). ${ }^{3}$

Anxiety disorder is a condition when someone felt an immense feeling of anxiety, where the feeling threatening their sense of peace and disturbing their ability to do any day-to-day activities. ${ }^{4}$ World Health 
Organization (WHO) data in 2015 conveyed that around 264 millions adults live with anxiety disorder all over the world. ${ }^{5}$ Global Burden of Disease Study also stated that in 2015, prevalence of anxiety disorder in Indonesia is $3.3 \%$ of the populations. ${ }^{5}$

The involvement of neurology in patients with lupus is called Neuropsychiatric Systemic Lupus Erythematosus (NPSLE). The manifestation of NPSLE may came out as a headache, mood disorder, anxiety disorder, or cognitive disorder. ${ }^{6}$ Neuropsychiatric SLE itself was found in $12.2-94.7 \%$ patients before, during, or after the making of diagnosis. According to the data that was screened at dr. Hasan Sadikin General Hospital Bandung, anxiety disorder stood in the fourth rank of the most frequent NPSLE after mood disorder, headache, and mononeuropathy. ${ }^{7}$

Anxiety felt by patients with lupus might be accompanied by autonomic disturbance. The autonomic disturbance by anxiety could be headache, perspiration, palpitation, difficulty of breathing, and restlessness. If the symptoms continue, it might affect patients' QOL. Quality of life in patients with SLE is a really important aspect, because it portrays how well they cope with daily struggle. It is crucial to note the dynamic of this aspect, especially because lupus is a chronic disease, thus the patients will have it throughout their whole life. Health, work and income, identity and independence, and social life and family life are important components to measure ones QOL. The result of QOL examination could be the guidence to determine the treatment of patients, and considered relevant for clinical practice. ${ }^{8}$ The knowledge about correlation between anxiety disorder and QOL in patients with SLE becomes important. The purpose of this study is to see the correlation between anxiety disorder and QOL in patients with SLE.

\section{Research Methods}

The study was conducted from June until September 2018 using primary data taken in the outpatient clinic of dr. Hasan Sadikin General Hospital. The subject of the study are patients diagnosed with SLE in Rheumatology Division Departement of Internal
Medicine dr. Hasan Sadikin General Hospital, suited with the study criterias. Patients who was diagnosed using SLE criteria from American Collage of Rheumatology in 1997, aged more than 18, and able to read and write were included in the study. Patients who are not willing to join the study, not filling out the questionnaire completely, and had other psychiatric disorder or drug abuse were excluded.

The assessment of QOL and anxiety disorder was done using Short From-36 (SF-36) and Zung SelfRating Anxiety Scale (Zung-SAS) questionnaire. The score for SF-36 questionnaire ranged from 1-100, meaning that the lower score obtained, the higher disabilities presents. Questionnaire was validated at University of Indonesia, showing a good result. 9 The score ranges of Zung-SAS are 20-80, which are 20-44 (normal), 45-59 (mild-moderate), 60-74 (severe), 75-80 (very severe or extra). Reliability test of Zung-SAS questionnaire was done at Universitas Pendidikan Indonesia, and also showing a good result (Cronbach a shows the value of 0.96). ${ }^{10}$

The minimum sample of this study was 46 patients. The data was taken using cross-sectional research design, and analyzed with Microsoft Excel 2011 and IBM $^{\circledR}$ SPSS $^{\circledR}$ Statistics version 20.0. The analysis of this study was conducted using Saphiro Wilk test to see the normality of the variables and Pearson or Spearman method to see the correlation between. Normality test shows that the distribution of this study variables are normal, so we could use the Pearson method. Pearson method was also choosen because both of the variables were numeric. The results of data analysis were shown in the form of tables.

Colletion of the data was done after we received an ethical clearance with number 520/UN6.KEP/EC/2018 from The Commission on Health Research Ethics Faculty of Medicine, University of Padjadjaran.

\section{Results}

Forty-seven patients diagnosed with SLE were included in the study. One patient was excluded because of the incomplete filling of questionnaire. 
Forty-six SLE patients were participated in this study.

Table 1 shows the demographic and characteristic of SLE patients by gender, age, SLE duration, and results of Zung-SAS and SF-36.

The result of Zung-SAS questionnaire and component from QOL of SF-36 were presented in table 2 and table $\mathbf{3}$, respectively.

Table 4 presents a significant negative correlation between anxiety disorder and QOL in patients with SLE, analyzed with Pearson method.

Table 1. Demography and Characteristic of Patients with Systemic Lupus Erythematosus

\begin{tabular}{ll}
\hline Variables & SLE Patients \\
\hline Gendera & $1(2,17 \%)$ \\
Male & $45(97.82 \%)$ \\
Female & \\
Age (year) & \\
$15-24$ & $7(15.21 \%)$ \\
$25-34$ & $17(36.95 \%)$ \\
$35-44$ & $13(28.26 \%)$ \\
$45-54$ & $8(17.39 \%)$ \\
$\geq 55$ & $1(2.17 \%)$ \\
SLE Duration (year)a & \\
$<1$ & $3(6.52 \%)$ \\
$1-5$ & $30(65.21 \%)$ \\
$>5$ & $13(28.26 \%)$ \\
Zung-SASb & $39.69 \pm 8.20$ \\
SF-36b & \\
Physical Component Summary (PCS) & $45.18 \pm 8.23$ \\
Mental Component Summary (MCS) & $47.11 \pm 9.78$ \\
\hline
\end{tabular}

aTotal (Percentage), bMean \pm Standard Deviation.

Table 2. Result of Zung-SAS Questionnaire

\begin{tabular}{lcc}
\hline Variables & Total (n) & Percentage (\%) \\
\hline Normal & 36 & $78.26 \%$ \\
Mild-moderate anxiety & 9 & $19.56 \%$ \\
Severe anxiety & 1 & $2.17 \%$ \\
Very Severe anxiety & 0 & 0 \\
$\quad$ Total & 46 & $100 \%$ \\
\hline
\end{tabular}

Table 3. The Result of Quality of Life Questionnaire (SF-36)

\begin{tabular}{ll}
\hline Variables & Mean \pm Standard Deviation \\
\hline Physical Functioning (PF) & $75.54 \pm 19.09$ \\
Physical Role Functioning (RP) & $60.05 \pm 24.24$ \\
Bodily Pain (BP) & $61.15 \pm 21.81$ \\
General Health Perception (GH) & $59.24 \pm 20.23$ \\
Vitality (VT) & $65.76 \pm 16.70$ \\
Social Role Functioning (SF) & $73.37 \pm 24.94$ \\
Emotional Role Functioning (RE) & $64.49 \pm 19.12$ \\
Mental Health (MH) & $78.26 \pm 29.15$ \\
\hline
\end{tabular}

Table 4. Correlation Between Anxiety Disorder and Quality of Life 


\begin{tabular}{lll}
\hline Variables & Zung-SAS & \\
& $\mathbf{r}$ & $\mathbf{P}$ \\
\hline Physical Component Summary (PCS) & -0.651 & 0.000 \\
Mental Component Summary (MCS) & -0.654 & 0.000 \\
\hline
\end{tabular}

\section{DISCUSSION}

The percentage of female with SLE (97.82\%) was higher than male (2.17\%). Systemic Lupus Erythematosus is an autoimmune disease that mostly affect woman, especially in productive age. The range of age mostly found was 25-34 years old (36.95\%), followed by the range of 35-44 years old (28.26\%). In a study conducted in Asia-Pasific, the prevalence of woman with lupus was ranged from 83 to $97 \%$ and the mean age was 25.7-34.5 years old. 3 The high lupus prevalence in reproductive age woman is believed to have an association with several hormones, such as estrogen, progesterone, testosterone, dehydrotestosterone, dan prolactin. These hormones is expected to cause the response from immune systems, like the stimulation of $\mathrm{T}$ and $\mathrm{B}$ cell, macrophage, and cytokine. ${ }^{11}$ The majority of SLE patients in this study was diagnosed with lupus for more than 1 year. The result of this study was in line with a study in AsiaPasific stating that the most prevalent duration of SLE ranged from 1.4-9.3 years old. ${ }^{3}$

Systemic Lupus Erythematosus is a multi-organ disease, with varied clinical manifestations. One of many clinical manifestation of SLE is an involvement with the nervous system. The involvement of this system will result in neurologic symptoms, with or without psychiatric symptoms. Anxiety disorder was placed at the fourth rank as neurologic disturbance that mostly happen in patients with SLE. ${ }^{7}$ Nine patients $(19.56 \%)$ in this study had a mild to moderate degree of anxiety, and 1 patient $(2.17 \%)$ had a severe degree of anxiety. This results were in line with previous research in Europe that showed a range of $17-75 \%$ for neuropsychiatric involvement in SLE patients. ${ }^{6}$

Patients with anxiety disorder have symptoms that will affect their QOL negatively in all domains. Anxiety disorder is found to have an association with a worsening in mental and physical function, compared to general population. ${ }^{12}$ The mean of QOL in patients with SLE itself was already low if it is to be compared with general population. ${ }^{13}$ Therefore, anxiety disorder in SLE patients became very important because if it remained to be unknown, it will impair their quality on living their life. A study in Canada identified that psychosocial variable (e.g.: social support) and behavior (e.g.: feeling of helplessness, the ability to handle ones disease) has a connection with QOL in patients with lupus. ${ }^{13}$ Quality of life in this study was assessed using SF-36 questionnaire, with the mean score of Physical Component Summary (PCS) and Mental Component Summary (MCS) slightly higher than study conducted in Canada. ${ }^{13}$

The coefficient correlation ( $\mathrm{r}$ ) from the correlation of Zung-SAS with PCS in this study was higher than the study conducted in China, while the $r$ of Zung-SAS with MCS was lower. This analysis showed that there were strong negative correlation between anxiety disorder and QOL in patients with SLE. Patients who have a higher degree of anxiety will have a lower QOL compared to patients with lower degree of anxiety. The study in China about the correlation between anxiety disorder and QOL in patients with SLE showed a similar result. ${ }^{14}$

Patients with anxiety disorder are found to have a decline in almost all aspects of QOL. ${ }^{15,16}$ The anxiety itself often associated with a reduction in ones productivity, disturbance in work, family, and social function, physical disability, and even mortality. A research North Carolina observed that after the demographic and other medical condition was controlled, anxiety disorder still had a significant association towards the limitation of patient's physical and mental function. 15 In the practice of medicine itself, anxiety disorder is commonly undetected, so it will be hard to give a suitable treatment in the first place. Study in Nigeria found that the detection prevalence of 
mental illness in primary health care facility was remarkably low, which was around $13.8 \%$. The early detection of anxiety disorder in patients becomes extremely important, because it could prevent the worsening of their QOL. An early intervention, such as cognitive therapy for the children and parenting training for the family, could be given to patients with anxiety disorder before it become severe, thus in the long run will lessen the burden of the disease and decrease the therapeutic cost.16,17 The distribution of anxiety disorder in SLE patients could be different in one study to another. Patient's educational level was not assessed, so the interpretation and knowledge of the patients about questions in the questionnaire might differ, therefore it is become the limitation of this study.

\section{CONCLUSION}

This study found a significant negative correlation of anxiety disorder and QOL in SLE patients. The analysis showed that the high degree of ones anxiety was in a parallel line with their low level of QOL, so it is important to do an early detection and prevention of anxiety disorder in SLE patients.

\section{Acknowledgement}

The researcher thanked The Lupus Study Group, Immunology Study Center, Faculty of Medicine, University of Padjadjaran for their help in providing the data of this study.

\section{REFERENCES}

1. Yazdany J, Dall'Era M. Definition and classification of lupus and lupus-related disorders. In: Wallace DJ, Hahn $\mathrm{BH}$, editors. Dubois' lupus erythematosus and related syndromes. $8^{\text {th }}$ Ed. Philadelphia: Saunders Elsevier; 2013. p. 1-7.

2. Hahn BH. Systemic lupus erythematosus. In: Longo DL, Kasper DL, Jameson JL, Fauci AS, Hauser SL, Loscalzo J, editors. Harrison's principles of internal medicine. 18 th Ed. United States of America: The McGraw-Hill Companies Inc.; 2012. p. 2724-35.

3. Jakes RW, Bae S-C, Louthrenoo W, Mok C-C, Navarra S V., Kwon N. Systematic review of the epidemiology of systemic lupus erythematosus in the asia-pacific region: prevalence, incidence, clinical features, and mortality. Arthritis Care Res (Hoboken). 2012;64(2):159-68.

4. Sadock BJ, Sadock VA, Ruiz P. Anxiety disorders. In: Pataki CS, Sussman N, editors. Synopsis of psychiatry: behavioral science/ clinical psychiatry. 11 th Ed. Philadelphia: Lippincott Williams \& Wilkins; 2015. p. 347-86.

5. World Health Organization. Depression and other common mental disorders: global health estimates. World Health Organization. Geneva; 2017.

6. Bertsias GK, Ioannidis JPA, Aringer M, Bollen E, Bombardieri S, Bruce IN, et al. EULAR recommendations for the management of systemic lupus erythematosus with neuropsychiatric manifestations: report of a task force of the EULAR standing committee for clinical affairs. Ann Rheum Dis. 2010;69(12):2074-82.

7. Putra SD, Ardisasmita MN, Hamijoyo L. Neuropsychiatric manifestation screening among systemic lupus erythematosus patients in hasan sadikin general hospital bandung. Indones $\mathrm{J}$ Rheumatol. 2017;9(2):18-22.

8. Bachen EA, Chesney MA, Criswell LA. Prevalence of mood and anxiety disorders in women with systemic lupus erythematosus. Arthritis Rheum. 2009;61(6):822-9.

9. Anindito B, Hidayat R, Koesnoe S, Dewiasty E. Validity and reliability of lupus quality of life questionnaire In patients with systemic lupus erythematosus in Indonesia. Indones $\mathrm{J}$ Rheumatol. 2016;8(2):38-44.

10. Heryana D. Correlation between the stage of anxiety and confidence on referee's performance while supervising a badminton match. Universitas Pendidikan Indonesia; 2012.

11. Maidhof W, Hilas O. Lupus: an overview of the disease and management options. 2012;37(4):240-7.

12. Beard C, Weisberg RB, Keller MB. Health-related quality of life across the anxiety disordersfindings from a sample of primary care patients. J Anxiety Disord. 2011;24(6):559-64.

13. Kuriya B, Gladman DD, Ibañez D, Urowitz MB. Quality of life over time in patients with systemic lupus erythematosus. Arthritis Care Res (Hoboken). 2008;59(2):181-5.

14. Shen B, Tan W, Feng G, He Y, Liu J, Chen W, et al. The correlations of disease activity, socioeconomic status, quality of life, and depression/anxiety in Chinese patients with systemic lupus erythematosus. Clin Dev Immunol. 2013;6.

15. Brenes GA. Anxiety, depression, and quality of life in primary care patients. J Clin Psychiatry. 
2007;9(6):437-43.

16. Soegaard HJ. Undetected common mental disorders in long-term sickness absence. Int $\mathrm{J}$ Family Med. 2012;9.
17. Morgan AJ, Rapee RM, Bayer JK. Prevention and early intervention of anxiety problems in young children: a pilot evaluation of cool little kids online. Elsevier. 2016;4:105-12. 